\title{
Advanced Automatic Control of Spatial Development Based on Crowdsourcing Platform
}

\author{
Irina AZAROVA ${ }^{1 *}$, Tatiana BEZVERKHNIUK ${ }^{1}$, Violetta VYSHNEVSKAYA ${ }^{1}$, \\ Anatoly RYBAK ${ }^{2}$, Pavel BALDUK ${ }^{3}$, Heorhii BALDUK ${ }^{3}$ \\ ${ }^{1}$ Odessa Regional Institute for Public Administration of the National Academy for Public Administration \\ under the President of Ukraine, 22 Genuezska Street, Odessa, 65009, Ukraine \\ azarovairene@gmail.com (*Correspondingauthor), tatiana.bezverkhniuk@gmail.com, viola@meta.ua \\ ${ }^{2}$ Odessa National Maritime University, 34 Mechnikova Street, Odessa, 65029, Ukraine \\ anatol166@ukr.net \\ ${ }^{3}$ Odessa State Academy of Civil Engineering and Architecture, 4 Didrikhson Street, Odessa, 65029, Ukraine \\ baldook.p@ogasa.org.ua,g.p.balduck@gmail.com
}

\begin{abstract}
The article describes the Crowdsourcing platform, which was developed to meet the challenges of sustainable spatial development. The platform allows users to formulate the problem of spatial development, submit proposals for solving the problem, choose the most optimal proposal by voting, plan project actions to implement the proposal, use various forms of crowd resources and provide public access to project reports. The proposed model provides a clear geographical location for both users and submitted problems, which ensures the formation of local communities that have common territorial interests to solve problems. The platform also makes it possible to use three kinds of participatory resources to implement proposals: labour, finance, and materials. At the end of the project, users can assess its success and its level of spatial problem resolution.
\end{abstract}

Keywords: Crowdsourcing, Spatial development information and communication systems, Project management.

\section{Introduction}

Recently, crowdsourcing has become a powerful tool for solving a wide range of variety tasks in different fields - from generating ideas for business development to solving complex political issues. One such area is spatial development, which can be defined as the process of preparation, adoption, and implementation in project format of decisions on space physical organization and providing on this basis the necessary spatial prerequisites for the fullest realization of the territorial system integrated potential (Kazmir, 2016).

Traditionally, project activities for Ukrainian spatial development are considered in the framework of vertical management structures carried out by local governments that manage development through a system of strategic planning (Spaliviero et al., 2019). However, due to such governance structures, it is quite difficult to effectively involve the public in spatial development processes. Crowdsourcing application in spatial development opens new perspectives on this issue. Consequently, this article is devoted to the study of crowdsourcing possibilities in spatial development, considered on the example of the crowdsourcing resource creation aimed to solving spatial problems in the project format. Section 2 of this study presents current directions for the use of crowdsourcing in spatial development, with some avenues for improvement. The third section describes the crowdsourcing platform model, including the initiation, implementation and completion of spatial development projects. Section 4 describes the results of the crowdsourcing platform practical operation, as well as an assessment of its effectiveness in solving spatial problems. Section 5 presents the conclusion and prospects for the development of the proposed research.

\section{Related Works}

Such a phenomenon as crowdsourcing has recently come to the attention of many researchers. Some existing definitions of crowdsourcing were analyzed by Estelles-Arolas \& GonzálezLadrón-De-Guevara (2012). The analysis of websites providing crowdsourcing services with some framework for systematic discussion, comparison and assessment of existing platforms were proposed by Mourelatos et al. (2016). Other researches (Papadopoulou \& Giaoutzi, 2014) investigated methods of integrating crowdsourcing with certain web technologies and GIS (geographic information systems) for spatial planning programs. The mechanisms of involving citizens in urban planning processes through crowdsourcing tools were also studied (Mueller et al., 2018). Scientists (Vogt \& Fröhlich, 2016) were focused on issues of providing immediate feedback to people engaged in participatory planning discussions including citizens, city 
administration, and entrepreneurs, through the Smarticipate platform.

Although these and some other studies reveal new aspects of stakeholder and communication management in spatial development projects, the comprehensive methodology for initiating and managing such projects remains out of focus in these studies.

Besides this, some scientists (Neto \& Santos, 2018) also paid their attention to the lack of standardization in the development of crowdsourcing projects and the relevance of developing a comprehensive methodology for managing crowdsourcing projects.

\section{The Crowdsourcing Platform for Spatial Development}

Based on the proposed definition (Erickson, 2011), crowdsourcing in the field of spatial development is the use of crowdsourcing resources through a crowdsourcing platform to achieve a clear result, such as solving the problem of developing a spatial system, including this problem formulation.

The crowdsourcing resources will mean all of the financial, perceptual, cognitive, or active (labor) abilities of a crowd. A spatial system will be considered a set of specific fundamental conditions created by man and nature within a certain territory. Crowdsourcing platform for spatial development will be considered as a system, software, or non-software, in which crowdsourcing is performed (Hosseini, 2014).
The problem of spatial development implies the deviation in the development of the spatial system identified by one or more target indicators detected and formalized with the help of a crowdsourcing platform. Indicator systems for smart cities (Poliakova \& Pozdniakova, 2019) or the sustainable development indicators mentioned above can be used as spatial development targets.

The spatial development problem solution will be considered as a successful implementation in the project format of the idea aimed at eliminating the corresponding deviations in the development of the spatial system.

Thus, the main characteristic features of crowdsourcing in spatial development are: (1) both the crowd and the solved spatial development problem are clearly localized; (2) local community members are interested in solving the spatial development problem; (3) the spatial development problem can potentially be solved by the efforts of the local community with / without the involvement of local authorities.

Crowdsourcing platform for spatial development (hereinafter - CPSD) is a software and hardware complex based on computer technology, consisting of interconnected information processing server, information storage server, input, and display devices, which users access via the Internet. The components of the CPSD and the scheme of their interaction are shown in Figure 1.

The main idea of this platform is that it will involve the widest possible range of stakeholders to pose the problem, plan, and implement management

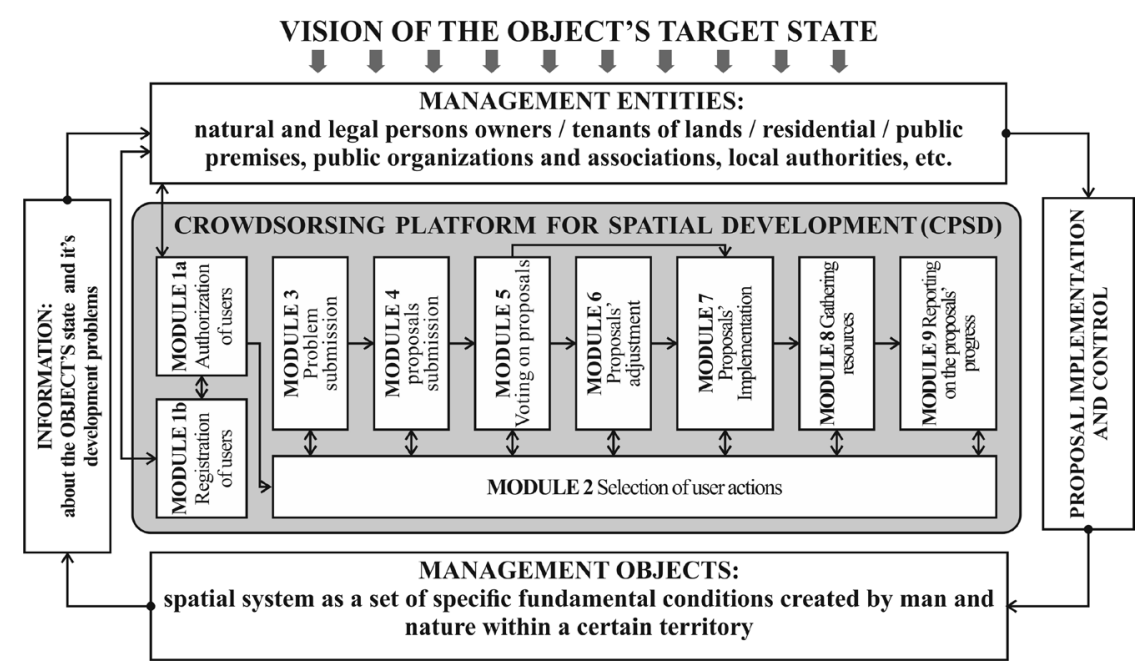

Figure 1. Components of the CPSD and the scheme of their interaction (adapted after Azarova \& Balduk, 2020)

https://www.sic.ici.ro 
decisions to eliminate it. All of such stakeholders will be the members of governance in spatial development in contrast to the traditional approach, where development management is solely a function of local government. Thus, in Ukraine, crowdsourcing is able to solve the traditional problem of the gap between strategic development planning and the real needs of local communities, which are not sufficiently involved by local authorities in the planning processes. Therefore, most of the strategic imperatives remain only on paper. At the same time, the actual problems of cities and other territories remain not only unresolved, but also informal (Svystovych, 2012).

The involvement of stakeholders will be carried out on the problem of territorial basis principle, which will form a local community of people who will live or work nearby and will be primarily interested in solving their problems.

The CPSD will also ensure the creation of information space for self-organization and the joint work of stakeholders at all stages of solving the problem - from setting the task to evaluating the results of the implementation of the decision.

The platform also provides for several forms of user participation in the implementation of the proposal, such as:

- participation in the form of labour;

- participation in the form of providing funds;

- participation in the form of allocation of material resources, including machinery and transport.

The proposed method also involves stakeholders on the CPSD basis to implement functions of planning and monitoring project activities for solving problems. In particular, it allows entities to establish and track the list of works, sequence, duration, and resource requirements.

The source of restrictions on the content of decision and the methods of their implementation, according to the concept proposed in the invention, are both the stakeholders' interests in their desired state of the object, as well as the current legislation.

The mathematical model of managing crowdsourcing spatial development projects is defined in more detail below.

\subsection{Initiation of the Crowdsourcing Spatial Development Projects Based on the CPSD}

Let all persons interested in the development of a certain area be marked as users of CPSD $-U$. They will include individuals who own and rent tenants of residential, public, and other premises, land, legal entities-entrepreneurs, public organizations and asSoCiations, representatives of local authorities, state supervisory authorities, etc. The set of these persons can be represented as a set of registered and unregistered users of CPSD:

$U=G$,

where $G$ - visitors who are not registered in the CPSD (unauthorized users);

$K_{j}$ - authorized users of the checkpoint, which are defined in the database of the checkpoint by parameters (2):

$$
K_{j}=\left(\log , \text { Pass }, L_{k}, e-\text { mail }, \text { Status }\right),
$$

where $\log$ is the login of the authorized user used to enter the checkpoint;

Pass - the password of the authorized user used to enter the checkpoint;

$j$ - number of authorized users of the checkpoint;

$L_{k}$ - target location of the authorized CPSD user (the place specified by the user on the map of the settlement which development problems interest the user);

$e$-mail- e-mail address of the authorized user used to receive information messages of the system;

Status - a variable that determines the type of authorized CPSD user and can take the values given in formula (3):

$$
\text { Status }=M \wedge K_{a} \wedge K b \wedge K_{b}^{\max } \wedge K_{r} \wedge K^{*}
$$

where $M-$ CPSD moderator;

$K_{a}$ - authorized CPSD user who submitted the problem $P_{m}$; 
$K_{b}$ - authorized CPSD user who posted a proposal $S_{n}$ aimed to solve the problem $P_{m}$

$K_{b}^{\max }$ - authorized CPSD user who posted the winning proposal $S_{n}^{\max }$ aimed to solve the problem $P_{m}$;

$K_{d}$ - authorized CPSD user who voted on the proposal $S_{n}$ aimed to solve the problem $P_{m}$;

$K_{r}$ - authorized CPSD user who offered resources to execute the proposal $S_{n}^{\max }$ aimed to solve the problem $P_{m}$;

$K^{*}$ - authorized CPSD user who took part in the implementation of the proposal $S_{n}^{\max }$, which was completed.

The parameters of the authorized user are set when he registers in the system in Module 1.

Authorization of the CPSD user is also carried out in Module 1, which verifies the coincidence of the authorization data entered by the user when logging in, with the records that were entered by him during registration in the system.

If the user successfully passes the authorization procedure, the transition to Module 2 is made, where he is asked to choose the necessary further action to work with the system.

In Module 2 the user's status is checked. Depending on this status, a list of user-allowed operations in the system is formed. Thus, unauthorized users $U$ have the right to review the list of submitted problems and proposals for their solution, receive reports on the progress of proposals implementation and collection of resources, as well as review the final reports on the proposal's completion. However, due to the impossibility of their identification in the system, such users do not have the right to submit new problems and solutions, vote for proposals and offer resources. These operations are only available to authorized $K_{j}$ users. $K_{a}$ users additionally have the opportunity to view the list of problems submitted by them. For $K_{b}$ users, there is a function of display and adjustment of the proposals submitted by them. Such a need may arise, for example, at the request of other authorized users to provide their support when voting. The ability to track user-submitted proposals that are in the implementation phase is provided for users $K_{b}^{\max }$. For the convenience of $K_{d}$ users, it is possible to track the proposals that were supported by them when voting. User $K_{r}$ is also offered to keep track of the proposals for which he offered resources.

Module 3 of the CPSD is designed to present the problem of spatial development identified by the user.

The detected problem can be denoted as $P_{m}$, where $m$ is the total number of submitted problems in the checkpoint. The problem is determined by the authorized user and the moderator by formula (4):

$P_{m}=\left(I_{P m}, L_{P m}, B_{P m}, H_{P m}, O_{P m}, t_{P m}, K_{a}\right)$,

where $I_{P m}$ is the name of the problem;

$L_{P m}$ - location of the problem (map location, GPS coordinates);

$B_{P m}$ - radius of coverage of the problem, meters;

$H_{P m}$ - horizon of urgency of the problem, days / weeks / months / years;

$O_{P m}$ - text description of the problem;

$t_{P m}$ - duration of posting the problem and submitting proposals for its solution, days (determined by the moderator);

$K_{a}$ - the authorized CPSD user who submitted the problem.

The description of the problem according to formula (4) is stored by the system in the appropriate database. When submitting a new problem, the system analyzes the coincidence of the submitted problem location and the target locations of authorized users of the checkpoint. If a match found, the relevant users will be notified by e-mail that a new problem has been reported at the user-specified target location. This provides an opportunity for users interested in the development of this location to form a local community on a territorial basis, which will be actively involved in solving a wide range of development problems of their neighborhood, housing, etc. For other users, a list of all submitted problems generated at their request in Module 2 is provided.

After the successful submission of the problem, Module 4 begins its work, in which proposals for solving the problem are submitted. 
For each proposal $S_{n}$ used to solve the problem $P_{m}$, the total number of such proposals $n$, is determined by the authorized user by formula (5):

$$
S_{n}=\left(P_{m}, K_{b}, I_{S n}, W_{x}, R_{W x}, T_{W x}, V_{W x}, E_{S n}\right),
$$

where $P_{m}$ is the problem, for which the solution is submitted;

$K_{b}$-authorized user who submitted a proposal $S_{n}$;

$I_{S n}-S_{n}$ proposal's name;

$W_{x}$ - list of works of the proposal $S_{n}$, (in text format), with the total number of $x$, pcs.;

$R_{W x}$ - resources for work $W_{x}$

$T_{W x}$ - time of performance of works $W_{x}$, hours;

$V_{W x}$ - sequence of works $W_{x}$ (predecessor task number);

$E_{S n}$ - the results to be obtained from the implementation of the proposal $S_{n}$.

Resources $R_{W x}$ for work $W_{x}$ are determined by formula (6):

$R_{W x}=\left(R_{W x}^{l a b}, R_{W x}^{\text {fin }}, R_{W x}^{m a t}\right)$,

where $R_{W x}^{l a b}$ - labor resources required to perform work $W_{x}$, man/hours;

$R_{W x}^{f i n}$ - cash resources required to perform work $W_{x}$, in local currency;

$R_{W x}^{\text {mat }}$ - material resources required to perform the work $W_{x}$, type of resource, and its quantity.

The submission of proposals is available to all authorized users during the posting time of $t_{P m}$ problem set by the moderator. If the posting time of the problem has expired and no suggestions for its solution have been received, the problem will be removed from the system due to its irrelevance for system users. For other issues, Module 5 voting for submitted proposals is further activated.

The main function of Module 5 is to provide support for management decision-making to select the best solution to solve the problem $S_{n}^{\max }$ which would satisfy the majority of users interested in the development of the territory. Users can set the "accept" or "reject" status for each submission. In case the user votes "decline", the system allows him to express in any form the conditions under which he could accept the proposal. The decision to adjust the proposal to ensure its exception is made by the user $K_{b}$, who receives appropriate notifications from the system about the content of the desired adjustments proposed by users during the voting. Proposals are adjusted in Module 6.

To select the optimal proposal for solving the problem for users $K$, formula (7) determines the share of $\gamma_{s n}$ "accept" votes for each of the proposals $S_{n}$ of the total number of voters for all the proposals on the problem $P_{m}$ :

$\gamma_{S n}=\frac{d}{Q_{S n}}$,

where $d$ is the number of $K_{d}$ users who voted "accept" for proposal $S_{n}$;

$Q_{P m}$ - the total number of voters for all the proposals submitted for the problem $P_{m}$.

At the end of the problem time, based on the values of $\gamma_{s n}$ calculated for each proposal, the proposal $S_{n}^{\max }$ is selected with the maximum value $\gamma_{S n} \rightarrow \max$. It will be accepted for further implementation under the guidance of user $K_{b}$, who submitted this proposal and appointed by the project leader if he seeks this.

This moment can be considered as the moment of initiation of the crowdsourcing project in the spatial development of the corresponding territory. The main characteristic feature and difference of the project management carried out with CPSD is the fully completion of planning processes during the project initiation phase. Nevertheless, the planning phase is the next phase after initiation according to project management standards.

\subsection{Implementation of the \\ Crowdsourcing Projects Based on the CPSD}

The implementation of the winning proposals based on the voting results is carried out according to the algorithm of Module 7. The implementation can 
be started after all the necessary resources have been collected.

The resource collection algorithm is in Module 8. Depending on the work specifics and the available resource opportunities of the users involved in the proposal implementation, users can participate in person as labor resources, provide money (financial resources), or involve their materials or mechanisms. User-selected resources $R_{W x}^{\text {prop }}$ can be defined in the system by formula (8):

$$
R_{W x}^{\text {prop }}=\left(Y_{W x}, N_{W x}, K_{r}\right) \text {, }
$$

where $Y_{W x}$ - the type of allocated resource (labor/ financial/material; the name of material resource);

$N_{W x}$ - the amount of allocated resource, units;

$K_{r}$ - an authorized user who allocated the resource $R_{W x}^{\text {prop }}$.

As soon as the system registers that all the necessary resources have been collected, the system translates the winning bid $S_{n}^{\max }$ into the implementation phase and notifies its manager $K_{b}^{\max }$ and the users who provided the resources.

Execution of works according to the proposal $S_{n}^{\max }$ is presented in the form of manual operation, with parallel periodic reporting for the project manager $K_{b}^{\max }$ about the works progress carried out according to the algorithm of Module 9. Reporting is submitted by the project manager $K_{b}^{\max }$ in terms of actual work performed, its actual duration, resources used, as well as the results obtained.

Reporting provides all users and visitors of the platform with an understanding of the processes underway, the direction of resource costs, and the degree of project management effectiveness.

\subsection{Completion of the Crowdsourcing Projects Based on the CPSD}

The moment of coinciding both the actual indicators of the project implementation with the planned ones, and obtained actual results with the expected ones the project implementation is completed with the formation of appropriate reporting for CPSD users. If the work on the proposal was performed in full, but it did not bring the expected results, the system established a discrepancy between the expected results and the chosen means of achieving them. The user $K_{b}^{\max }$ is asked to review his proposal and make the necessary adjustments to the planned actions in Module 6. Then the resource collection under Module 8 is initiated again and the proposal is implemented by taking into account the changes under Module 7. The project cycle repeats until the problem is solved and the expected results are achieved.

To establish the fact of obtaining the expected result of the project, users can vote on this issue. Although an additional method may be the analysis of relevant indicators and indicators of development, according to the authors of the study, preference should be given to user assessments of the results obtained and the degree of the solution of a particular problem.

Successful completion of the project brings all its participants and project manager an appropriate award in their profile, which will increase their status and skills in crowdsourcing projects. Officials involved in project implementation as public relations tools or indicators of their interaction effect with the public can use such statuses. Other users may post such statuses on SoCial media or professional profiles and CVs.

Information about completed projects remains on the platform so that during the implementation of the next similar projects, their manager could use the necessary information and contacts of such a project.

Based on such a crowdsourcing platform, the problems of spatial development in any sphere and on any scale can be solved: from eliminating road surface defects in the yard of an apartment building to creating a specialized network of bicycle and pedestrian paths in the city. Due to the lack of territorial restrictions for the problems presented on the platform, there is a unique opportunity to solve these problems without taking into account the boundaries of administrative units and settlements. This is very important for the problems of urban agglomerations that go beyond the administrative boundaries of a particular settlement. The platform will also help to unite efforts to solve common problems on the border of several territorial units, which are traditionally considered by each of them in isolation. 
Depending on the scope and scale of the problems, some of them will be addressed as part of infrastructure enterprises' operational activities to serve the city. At the same time, the citizens of the city will also be able to take part in these operational processes, providing their resources and exercising control over these activities.

Other issues may need to be addressed at the strategic or national strategic planning level. In this case, to ensure an effective solution to the problem, it will be necessary to involve representatives of local authorities as active users of the platform. However, today in Ukraine there is no such mechanisms of interaction between the authorities and the public provided by law.

\section{The Practical Results of the CPSD Activity}

To implement the proposed model with the technical support of an educational non-profit organization, a specialized CPSD was created to address the problematic issues of development of the Ukrainian city of Odesa $<$ https://lopata.pp.ua $>$. This information resource aimed to improve the quality of the urban environment by optimizing the processes of urban development management. This information resource was designed, built and implemented using the lifecycle methodology described by Filip (2012).

The created CPDS comprises of 9 main modules.

Spatial localization of problems and registered users was carried out based on the "Mapbox Maps SDK and GL JS" application from the "Mapbox", which offered a convenient alternative to the wellknown "Google Maps" service. The application offers non-commercial clients the use of "Open Street Map" maps to integrate personalized vector maps for web applications.

The user interface of the platform was implemented from the app "Vue.js" - "JavaScript" - an open source framework (You, 2020).

The platform also uses the "Cloud Functions" provided by "Firebase". This is a server-free framework, allowing the user to automatically run backend code in response to events triggered by "Firebase" features and HTTPS requests. It is used to verify the expiration date of the problems and to securely view users' phones when they need them.
The next section of "Firebase feature" - "Cloud Storage" - is used for storing and serving user-generated content and static files, such as problems' images, solutions' images, and fonts.

"Google Analytics", a free analytics service provided by "Google", is used to obtain information about how users find and use the platform. With "Google Analytics", the conversion on the forms in which the user visits the site and adds a problem or a solution is monitored.

The development of this crowdsourcing resource took about six months. The project team consisted of three people: programmer, designer and project manager. In early March 2020, a beta version of the crowdsourcing platform was launched.

Over the next 5 months 1,632 users visited the platform. In total, 117 users were registered and submitted 31 city problems. Among the most frequently submitted problems are the poor condition of houses, roads, streets, parks, and the lack of car parking, playgrounds and sports grounds, public transport problems. 13 solutions of posted problems were also added. Two of the posted problems have already been successfully solved.

Based on these results, to analyze the effectiveness of using crowdsourcing in solving problems of spatial development, it will not be enough to simply compare the features of the created platform with similar resources (Ciurea \& Filip, 2019). It is necessary to assess both the actual willingness of local community members to participate in spatial development activities through the created CPSD and the effectiveness of this resource in solving local spatial development problems.

Thus, the effectiveness of CPSD was assessed based on the following evaluation indicators (Azarova \& Balduk, 2020):

$\mathrm{P}$ - population of the region / territorial unit, people;

$\mathrm{RB}$ - the indicator of the budget revenue of the region / territorial unit, expressed in local currency units;

$\mathrm{U}$ - the number of registered users of the platform, people;

PP - the number of submitted problems on the platform for a certain period, units; 
SSP - the number of successfully resolved problems (completed projects) for the same period, units;

PB - budgets of completed projects, local currency units.

Based on the data obtained, the system of Ukrainian crowdsourcing platforms performance indicators was proposed.

To assess the willingness of local community members to participate in solving spatial development problems on crowdsourcing platforms, Crowdsourcing Activity Rate index (CAR, \%) was calculated according to formula (9) as the ratio of registered platform users to the total population of the region:

$$
C A R=\frac{U}{P} 100 \%
$$

As an indicator of population's involvement in the processes of generating ideas for solving specific problems of spatial development was chosen the Coefficient of Project Activity (CPA, \%), which means the ratio of the number of problems posted on the platform to the number of population in the region according to formula (10):

$C P A=\frac{P P}{P} 100 \%$

To understand the level of realism and feasibility of user-proposed projects, a Project Performance
Ratio (PPR, \%) was introduced. It is obtained by formula (11) as the ratio of the number of completed projects to the total number of proposals submitted for the period under review:

$P P R=\frac{S S P}{P P} 100 \%$

The scale of solved problems implemented on the crowdsourcing platform may be estimated based on the Budget Capacity of Completed Projects (BCCP, \%), calculated as the ratio of the sum of completed projects budgets to the state budget revenue of such region according to formula (12):

$$
B C C P=\frac{P B}{R B} 100 \%
$$

The calculated values of the efficiency indicators of the created crowdsourcing platform are presented in Table 1.

Thus, the following results of the platform operation were obtained. The coefficient of project activity of the created platform is $24 \%$ of the average for Ukraine. The crowdsourcing activity index is $31 \%$, and the project implementation rate is about $26 \%$. These indicators shows the insufficient efficiency of the created crowdsourcing resource in comparison with similar ones. It is expected that the promotion of this platform will significantly increase site traffic and its effectiveness.

Table 1. Evaluation of the effectiveness of the crowdsourcing platform in solving the development problems of

\begin{tabular}{|c|c|c|c|c|c|c|c|c|}
\hline \multirow[t]{2}{*}{ 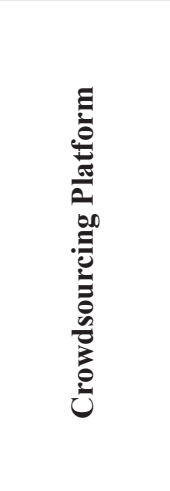 } & 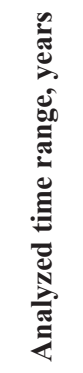 & 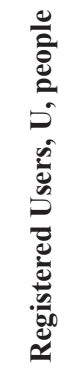 & 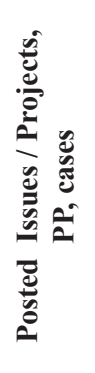 & 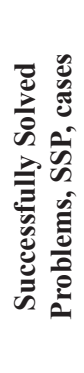 & 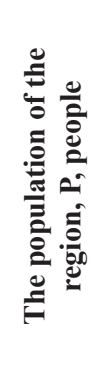 & 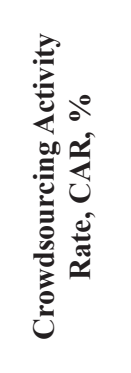 & 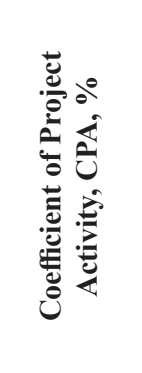 & 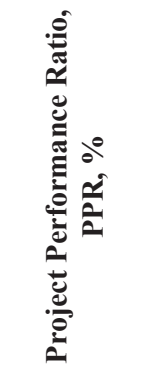 \\
\hline & $\mathrm{D}$ & $\mathrm{U}$ & PP & SSP & $\mathrm{P}$ & $\begin{array}{l}C A R= \\
\frac{U}{P} \times 100\end{array}$ & $\begin{array}{l}C P A= \\
\frac{P P}{P} \times 100\end{array}$ & $\begin{array}{l}P R= \\
\frac{S S P}{P P} \times 100\end{array}$ \\
\hline $\begin{array}{c}\text { https:// } \\
\text { lopata.od.ua/ }\end{array}$ & 2020 & 280,8 & 74,4 & 4,8 & 997766 & 0,028 & 0,007 & 6,45 \\
\hline \multicolumn{6}{|c|}{ On average in Ukraine*: } & 0,091 & 0,031 & 25,14 \\
\hline \multicolumn{6}{|c|}{ On average in Europe*: } & 3,535 & 0,073 & 17,73 \\
\hline
\end{tabular}
the city of Odessa (adapted after Azarova \& Balduk, 2020)

* data can be obtained on request to azarovairene@gmail.com 


\section{Conclusion}

In the present study, the possibility and feasibility of implementing certain functions of managing spatial development projects based on crowdsourcing has been successfully confirmed. A model for managing crowdsourcing projects through the CPSD has also been proposed.

Based on the proposed model, the solution of spatial development issues was ensured with the participation of crowdsourcing resources.

Thus, the CPSD allows not only the involvement of a wide range of stakeholders in spatial development, including the processes of strategic planning, financing, and implementation of activities for the development of various territories but also the delegation to the final beneficiaries of some management functions and responsibilities for spatial development trends in this area. The proposed model allows using a wide range of crowdsourcing tools to increase the efficiency of the spatial development system and the implementation of relevant projects, both in urban and rural regions.

The clear geographical localization for both users and submitted problems ensures the formation of local communities that have common territorial interests to solve problems. CPSD also allows users

\section{REFERENCES}

Anon, (2021). LOPATA Platform for Solving Urban Problems. Available at: <https://lopata.pp.ua/>, last accessed: April 22th, 2021.

Azarova, I. \& Balduk, G. (2020). Solving the problems of sustainable spatial development of Ukrainian cities based on the crowdsourcing principles, Scientific notes of Taurida National V.I. Vernadsky University. Series: Economy and Management, 32(71), No. 1, 106-122. DOI: https://doi.org/10.32838/2523-4803/70-5-17

Ciurea, C. \& Filip, F. G. (2019). Collaborative Platforms for Crowdsourcing and Consensusbased Decisions in Multi-Participant Environments, Informatica Economica, 23, 5-14. DOI: 10.12948/ issn14531305/23.2.2019.01

Erickson, T. (2011). Some thoughts on a framework for crowdsourcing. In Materials of CHI 2011: Workshop on Crowdsourcing and Human Computation (pp. 1-4).

Estelles-Arolas, E. \& González-Ladrón-De-Guevara, F. (2012). Towards an integrated crowdsourcing to use three types of crowd resources to implement proposals: labor, financial, and material.

The implementation of the proposed model of a spatial development management system based on the CPSD proved the practical feasibility and value of the model for solving spatial development problems, which is confirmed by the obtained results of the resource work.

On the basis of the platform, various problems of territorial development can be solved, including improving the quality of the urban environment, determining strategic priorities for further development, studying public opinion and needs in the field of spatial development. The resource can also become the basis for creating a long-term and productive partnership between citizens and authorities in strategic planning and implementation of development strategies, which is a particularly pressing issue for Ukraine. As a true crowdsourcing resource, the platform can also be a source of innovation in spatial development and other neighboring industries.

Improving the efficiency of this resource by expanding the number of CPDS users and ongoing projects can be ensured by raising awareness and involvement of residents in the process of managing the development of their city.

definition, Journal of Information Science, 32(2), 189200. DOI:10.1177/0165551512437638

Filip, F. G. (2012). A Decision-Making Perspective for Designing and Building Information Systems, International Journal of Computers Communications \& Control, [S.1.], 7(2), 264-272. DOI:10.15837/ijccc.2012.2.140

Hosseini, M., Phalp, K., Taylor, J. \& Ali, R. (2014). The four pillars of crowdsourcing: A reference model. In Proceedings of the 2014 IEEE Eighth International Conference on Research Challenges in Information Science (RCIS), (pp. 1-12).

Kazmir, L. P. (2016). Peculiarities of spatial development planning of metropolitan subregions. Scientific note. Institute for Regional Studies named after M. I. Dolishny of the National Academy of Sciences of Ukraine.

Mourelatos, E., Tzagarakis, M. \& Dimara, E. (2016). A review of online crowdsourcing platforms, SouthEastern Europe Journal of Economics, 1, 59-74. 
Mueller, J., Lu, H., Chirkin, A., Klein, B. \& Schmitt, G. (2018). Citizen Design Science. A strategy for crowd-creative urban design, City, 72(A), 181-188.

Neto, F. A. \& Santos, C. A. S. (2018). Understanding crowdsourcing projects: A systematic review of tendencies, workflow, and quality management, Information Processing \& Management, 54(4), 490-506.

Papadopoulou, C.-A. \& Giaoutzi, M. (2014). Crowdsourcing as a Tool for Knowledge Acquisition in Spatial Planning, Future Internet, 6(1), 109-125.

Poliakova, O. \& Pozdniakova, A. (2019). A methodological approach to evaluating the progress of the implementation of the smart city concept in Ukrainian cities, The Problems of Economy, 39(1), 74-82.

Spaliviero, M., Boerboom, L., Gibert, M., Spaliviero, G. \& Bajaj, M. (2019). The Spatial Development
Framework to facilitate urban management in countries with weak planning systems, International Planning Studies, 24(3-4), 235-254.

Svystovych, M. (2012). Strategic planning in Ukraine: history and problems of implementation, Bulletin of the National Academy of Public Administration under the President of Ukraine, 2, 46-53.

Vogt, M. \& Fröhlich, P. (2016). Understanding cities and citizens: Developing novel participatory development methods and public service concepts. In Proceedings of 21 st International Conference on Urban Planning, Regional Development and Information SoCiety (pp. 991-995).

You, E. (2020). The Progressive JavaScript Framework. Available at: <https://vuejs.org/>, last accessed: April 22th, 2021. 